\title{
Efectos de fertilización potásica en el cultivo de pimiento (Capsicum annum L.) VAR: HÍBRIDO NATHÁLIE
}

\author{
Effects of potassium fertilization in the crop of pepper (Capsicum annum L.) \\ VAR: HYBRID NATHALIE
}
Efeitos da fertilização com potássio nas culturas de pimenta (Capsicum annum L.) VAR: NATHALIE HYBRID

\author{
- Liz Dolorez Toñanez Pavón \\ toñanezliz@gmail.com \\ Código ORCID: 0000 - 0002-7426-926X
}

\author{
- Juan Alberto Bottino Fernández \\ jabfer@yahoo.com.ar \\ Código ORCID: 0000 - 0003-0404-4169
}

Ximena Judith Galeano Graupera
ximenajudithg@gmail.com
Código ORCID: 0000 - 0001-9508-8268

Facultad de Ciencias Agropecuarias y Desarrollo Rural - UNP, Paraguay

Artículo recibido 24 de noviembre 2020 / Arbitrado y aceptado 17 de diciembre 2020 / Publicado 04 de enero 2021

\section{RESUMEN}

La investigación denominada Efectos de la aplicación de diferentes niveles de fertilización potásica en el rendimiento y la calidad de frutos en el cultivo de pimiento (Capsicum annum L.) Var: Híbrido Nathalie, en el distrito de Pilar, año agrícola 2017/2018, fue presentada a la Facultad de Ciencias Agropecuarias y Desarrollo Rural, Universidad Nacional de Pilar. Investigación de tipo experimental, enfocado a la evaluación de diversos parámetros en la producción de pimiento de la variedad Híbrido Nathalie, con aplicación de diferentes niveles de fertilización potásica, los resultados obtenidos valorar la influencia de dichos tratamientos en la calidad y el rendimiento del cultivo de pimiento. Las dosis utilizadas fueron las siguientes $50,75,100,125$, y $150 \mathrm{~kg} / \mathrm{ha}$, incluyendo un testigo el cual fue el tratamiento sin aplicación de fertilizante potásico, contando así con seis tratamientos y cinco repeticiones distribuidos en bloques al azar. Dicho análisis se comprobó con pruebas estadísticas, análisis de la varianza y la comparación de medias a través de la prueba de Duncan al 5\%. Respecto a la calidad, las medias de longitud, diámetro y grosor de pared del fruto no presentaron grandes diferencias, destacando que el T4 (100 Kg/ha) siempre presentó un nivel superior entre los tratamientos, y en cuanto al rendimiento el análisis de varianza arrojó que no existe diferencia significativa entre los tratamientos, sin embargo, el T4 tuvo el mayor rendimiento con $55.225 \mathrm{Kg} / \mathrm{ha}$ donde la comparación de medias indicó que presentó diferencia significativa sobre el testigo.

Palabras clave: Pimiento; Fertilización; Rendimiento; Calidad
ABSTRACT

The investigation called Effects of the application of different levels of potassium fertilization on the yield and quality of fruits in the cultivation of pepper (Capsicum annum L.) Var: Híbrido Nathalie, in the district of Pilar, agricultural year $2017 / 2018$, was presented to the Faculty of Agricultural Sciences and Rural Development, National University of Pilar. Experimental research, focused on the evaluation of various parameters in the production of the hybrid Nathalie variety pepper, with the application of different levels of potassium fertilization, the results obtained assess the influence of said treatments on the quality and yield of the crop of pepper. The doses used were the following 50,75 , 100,125 , and $150 \mathrm{~kg} / \mathrm{ha}$, including a control which was the treatment without application of potassium fertilizer, thus having six treatments and five repetitions distributed in random blocks. This analysis was verified with statistical tests, analysis of variance and the comparison of means through the Duncan test at 5\%. Regarding quality, the means of length, diameter and wall thickness of the fruit did not show great differences, highlighting that the $\mathrm{T} 4(100 \mathrm{Kg} / \mathrm{ha})$ always presented a higher level between the treatments, and in terms of yield the analysis of variance showed that there is no significant difference between the treatments, however, the T4 had the highest yield with $55,225 \mathrm{Kg} /$ ha where the comparison of means indicated that it presented a significant difference over the control.

Key words: Pepper; Fertilization; Yield; Quality
RESUMO

De modo a dar cumplimiento a los requisitos exigidos para acceder al título de Ingeniero Agropecuario, se realiza la investigación denominada Efectos de la aplicación de diferentes niveles de fertilización potásica en el rendimiento y la calidad de frutos en el cultivo de pimiento (Capsicum annum L.) Var: Híbrido Nathalie, en el distrito de Pilar, año agrícola 2017/2018 presentado a la Facultad de Ciencias Agropecuarias y Desarrollo Rural, Universidad Nacional de Pilar. Esta investigación fue del tipo experimental, enfocado a la evaluación de diversos parámetros en la producción de pimiento de la variedad Híbrido Nathalie con aplicación de diferentes niveles de fertilización potásica, con el fin del análisis de los resultados obtenidos valorar la influencia de dichos tratamientos en la calidad y el rendimiento del cultivo de pimiento. Las dosis utilizadas fueron las siguientes $50,75,100,125$, y $150 \mathrm{~kg} / \mathrm{ha}$ incluyendo un testigo el cual fue el tratamiento sin aplicación de fertilizante potásico, contando así con seis tratamientos y cinco repeticiones distribuidos en bloques al azar. Dicho análisis se comprobó con pruebas estadísticas, análisis de la varianza y la comparación de medias a través de la prueba de Duncan al 5\%. Respecto a la calidad, las medias de longitud, diámetro y grosor de pared del fruto no presentaron grandes diferencias, destacando que el T4 (100 Kg/ha) siempre presentó un nivel superior entre los tratamientos, y en cuanto al rendimiento el análisis de varianza arrojó que no existe diferencia significativa entre los tratamientos, sin embargo, el T4 tuvo el mayor rendimiento con $55.225 \mathrm{Kg} /$ ha donde la comparación de medias indicó que presentó diferencia significativa sobre el testigo.

Palavras-chave: Pimiento; Fertilización; Rendimiento; Calidad 


\section{INTRODUCCIÓN}

La influencia que tiene los diferentes niveles de fertilización potásica en el rendimiento y la calidad de frutos en el cultivo de pimiento (Capsicum annum L.), la variedad cultivada para la experimentación fue Nathalie, un híbrido caracterizado por su fruta alargada terminada en punta son muchos de los datos que se muestran en este articulo

Con respecto al mercado, esta hortaliza presenta los primeros lugares de preferencia de consumo junto con la cebolla y el tomate, destacando su comercialización, los consumidores se encuentran especialmente en los restaurantes y en los hogares donde las amas de casas lo utilizan diariamente en la gastronomía donde aprovechan el fruto en fresco como también en conservas.

Según el Departamento de Estadísticas/DCEA, con datos de Encuestas Agrícolas, Sub Centros de la DCEA y DEAg/MAG, la superficie cultivada de esta hortaliza en el Paraguay en la Región Oriental en el año agrícola 2015/16 fue de 690 ha, con una producción de 6.900Tn y un rendimiento promedio de $10.000 \mathrm{~kg} / \mathrm{ha}$. Las principales zonas de producción se encuentran en los departamentos de San Pedro, Caaguazú, Itapúa y Central.

Esta hortaliza se adapta perfectamente a la región climática del país siendo una posible alternativa de renta para el productor de la zona, quienes no realizan los trabajos necesarios para una producción en cantidad, continuidad y calidad, tres factores fundamentales para satisfacer y abastecer el mercado local. Para cumplir con estos requerimientos, se requiere de técnicas e infraestructura adecuada con la cual se obtendría un rendimiento que beneficiaría de sobremanera al productor y así también al consumidor.

El trabajo de investigación se desarrolló en la ciudad de Pilar Departamento de Ñeembucú, ubicado en el $\mathrm{B}^{\circ}$ Crucecita. Dicha experimentación se basó en la aplicación de diferentes niveles de fertilización potásica para la determinación del nivel más adecuada, que alcance el mayor rendimiento y la mejor calidad de frutas en el cultivo de pimiento, sabiendo que los nutrientes minerales cumplen importantes funciones en la planta siendo el potasio uno de los nutrientes esenciales para obtener frutos de calidad y con un alto rendimiento ya que actúa en las diferentes etapas de desarrollo de la planta.

Es estudio tuvo como propósito evaluar el rendimiento y la calidad de frutos en el cultivo de pimiento (Capsicum annum L.) Var: Híbrido Nathalie con la aplicación de diferentes niveles de fertilización potásica, en el distrito de Pilar, año agrícola 2017/2018; determinar el nivel de fertilización potásica aplicado que brinda mayores rendimientos en el cultivo de pimiento Var: Nathalie; establecer el nivel fertilización potásica más adecuado que brinda la mejor calidad de frutos de pimiento Var: Nathalie; determinar la relación de los costos de producción y el beneficio neto obtenido a partir de diferentes niveles de fertilización potásica por unidad de superficie, en el cultivo de pimiento Var: Nathalie. En donde se evaluó C. Hipótesis $\mathbf{H}_{\mathrm{A}}$ : El rendimiento y la calidad de frutos en el cultivo de pimiento (Capsicum annum L.), Var: Híbrido Nathalie son características variables dependientes del nivel de fertilización potásica. $\mathbf{H}_{\mathbf{0}}$ : $\mathrm{El}$ rendimiento y la calidad de frutos en el cultivo de pimiento (Capsicum annum L.), Var: Híbrido Nathalie son características constantes e 
independientes del nivel de fertilización potásica.

\section{MATERIALES Y MÉTODOS}

El trabajo de investigación se desarrolló en la parcela perteneciente a la Sra. Leonida Antola de Beconi ubicado en el $\mathrm{B}^{\circ}$ Crucecita de la ciudad de Pilar, Departamento de Ñeembucú-Paraguay, cuyas coordenadas geográficas son $26^{\circ} 52^{\prime}$ Latitud Sur y $58^{\circ} 17^{\prime}$ Longitud Oeste.

Enfoque de la investigación Cuantitativa por lo común este enfoque se utiliza primero para descubrir y refinar preguntas de investigación. A veces, pero no necesariamente, se prueban hipótesis. Con frecuencia se basa en métodos de recolección de datos sin medición numérica, como las descripciones y las observaciones. (1).

Para probar las hipótesis ya establecidas con estos dos enfoques se midió el rendimiento y la calidad de frutos en el cultivo de pimiento utilizando métodos estadísticos para probar los efectos de los niveles de fertilización potásica sobre las variables analizadas.

El diseño utilizado en la experimentación fue la Distribución en Bloques al Azar esta distribución de los tratamientos es la de mayor uso en el diseño de experimentos y tiene grandes ventajas cuando el número de tratamientos no excede de 15 y cuando es posible agrupar las unidades experimentales en estratos o bloques uniformes, de tal manera que la variabilidad entre unidades experimentales es mínima, aun cuando la variación entre estratos o bloques sea alta (2).

Fue descriptivo Méndez (3), indica que la investigación descriptiva utiliza criterios sistemáticos que permiten poner de manifiesto la estructura de los fenómenos en estudio, además ayuda a establecer comportamientos concretos mediante el manejo de técnicas específicas de recolección de información. Así, el estudio descriptivo identifica características del universo de investigación, señala formas de conducta y actitudes del universo investigado, descubre y comprueba la asociación entre variables de investigación.

En opinión de Arias (4), los estudios descriptivos permiten medir de forma independiente las variables, aun cuando no se formule hipótesis alguna, pues éstas aparecen enunciadas en los objetivos de la investigación, de allí que el tipo de investigación esté referido a escudriñar con cuanta profundidad se abordará el objeto, sujeto o fenómeno a estudiar.

Según Tamayo y Tamayo (5) la población es la totalidad del fenómeno a estudiar en donde la unidad de población posee una característica común, la cual se estudia y da origen a los datos de investigación, y la muestra es cuando seleccionamos algunos de los elementos con la intención de averiguar algo sobre la población de la cual están tomadas.

Por tanto, en esta investigación la población comprende la totalidad de plantas de pimiento en cada uno de los tratamientos, repeticiones y efecto bordes, sumando un total de 420 plantas de pimiento y la muestra constituye la totalidad de las plantas establecidas dentro del área útil $\left(4 \mathrm{~m}^{2}\right), 8$ plantas por tratamiento sumando un total de 240 plantas evaluadas.

\section{Detalle de los tratamientos}

Diseño de Bloques al Azar. El diseño estuvo constituido con seis tratamientos y cinco repeticiones o bloques. 
- La superficie de cada unidad experimental de $5 \mathrm{~m}^{2}$ ( $5 \mathrm{~m}$ de largo por $1 \mathrm{~m}$ de ancho)

- La distancia entre cada uno de los bloques es de $1 \mathrm{~m}$ y de cada número de unidades experimentales es de $0,60 \mathrm{~m}$.

Tamaño total de la parcela experimental: la superficie del ensayo fue de $246,24 \mathrm{~m}^{2}$. (34,2 $\mathrm{m}$ de largo y 7,2 $\mathrm{m}$ de ancho) esto incluyendo los camineros y las parcelas de efecto borde (Ver la figura $\mathrm{N}^{\circ} 1$ ).

\section{Referencias de los tratamientos a aplicar}

La fertilización mineral recomendada a partir del análisis de suelo es de 120-120-100 $\mathrm{kg} / \mathrm{ha}$ de N, P2O5 y K20, respectivamente.
Los tratamientos utilizados fueron los siguientes:

- Tratamiento $\mathrm{N}^{\circ} 1=0 \mathrm{~kg} / \mathrm{h}$ de $\mathrm{K}$.

- Tratamiento $\mathrm{N}^{\circ} 2=50 \mathrm{~kg} / \mathrm{h}$ a de $\mathrm{K}$.

- Tratamiento $\mathrm{N}^{\circ} 3=75 \mathrm{~kg} / \mathrm{h}$ a de $\mathrm{K}$.

- Tratamiento $\mathrm{N}^{\circ} 4=100 \mathrm{~kg} / \mathrm{ha}$ de $\mathrm{K}$.

- Tratamiento $\mathrm{N}^{\circ} 5=125 \mathrm{~kg} / \mathrm{ha}$ de K.

- Tratamiento $\mathrm{N}^{\circ} 6=150 \mathrm{~kg} / \mathrm{ha}$ de $\mathrm{K}$.

La fertilización potásica fue realizada con Cloruro de Potasio (0-0-60). El mismo se aplicó $20 \%$ de base y el $80 \%$ de cobertura a partir de la floración aplicando cada 10 días.

\section{Diseño y dimensiones del experimento}

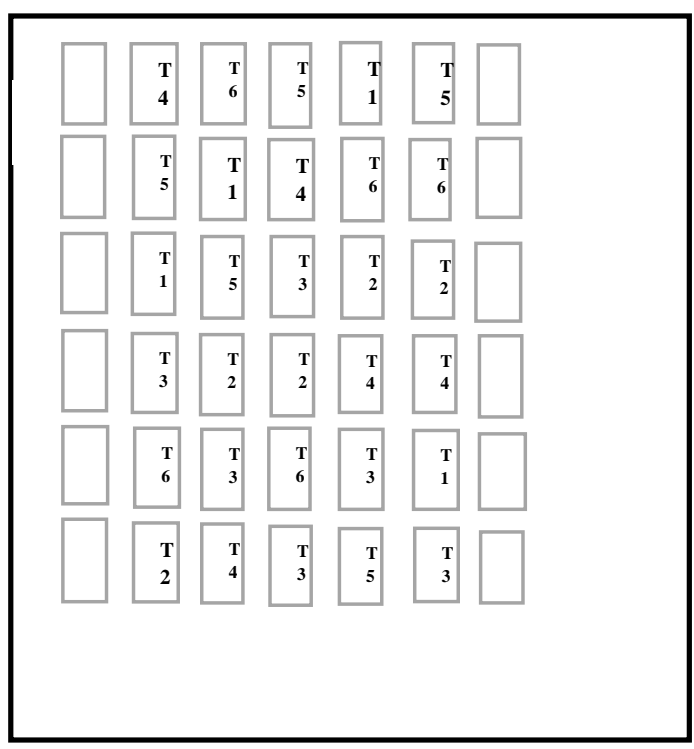

Dimensiones: $1 \mathrm{~m} \times 1 \mathrm{~m}$ 


\section{Técnica utilizada}

La técnica de recolección de datos se basó en la medición de las características según los indicadores mediante el cual se recogió datos sobre la muestra y se registraron en planillas: el peso promedio de frutos en gramos, cantidad de frutos por planta, longitud y diámetro de frutos, espesor de pared de los frutos y mediante la observación directa la forma comercial y coloración de los frutos para luego ser debidamente procesados.

\section{Descripción de actividades}

1. Análisis del suelo

2. Pre-germinación de semillas en estufa (04-08-17).

3. Repique de semillas pre-germinadas en bandejas (07-08-17).

4. Preparación del terreno (21-08-17).

5. Marcación del terreno (26-08-17).

6. Preparación de camellones (28-08-17) (29-08-17).

7. Aplicación de estiércol vacuno (04-0917).
8. Instalación de sistema de riego por goteo (06-09-17).

9. Aplicación de fertilizante de base (11-0917).

10. Colocación de cobertura de suelo (mulching) (12-09-17).

11. Trasplante de plantines de pimiento (1609-17).

12. Pulverización.

13. Fertilización de cobertura (urea).

14. Entutorado (21-10-17) -(22-10-18).

15. Aclareo de flores, desbrotes y eliminación de hojas.

16. Fertilización de cobertura (cloruro de potasio).

17. Instalación de malla media sombra (1411-2017).

18. Cosecha.

19. Pesaje y mediciones.

\section{RESULTADOS Y DISCUSIÓN}

En este apartado iniciamos con las evidencias obtenidas mediante el análisis de los resultados que dieron paso en lo que fue la construcción del estudio.

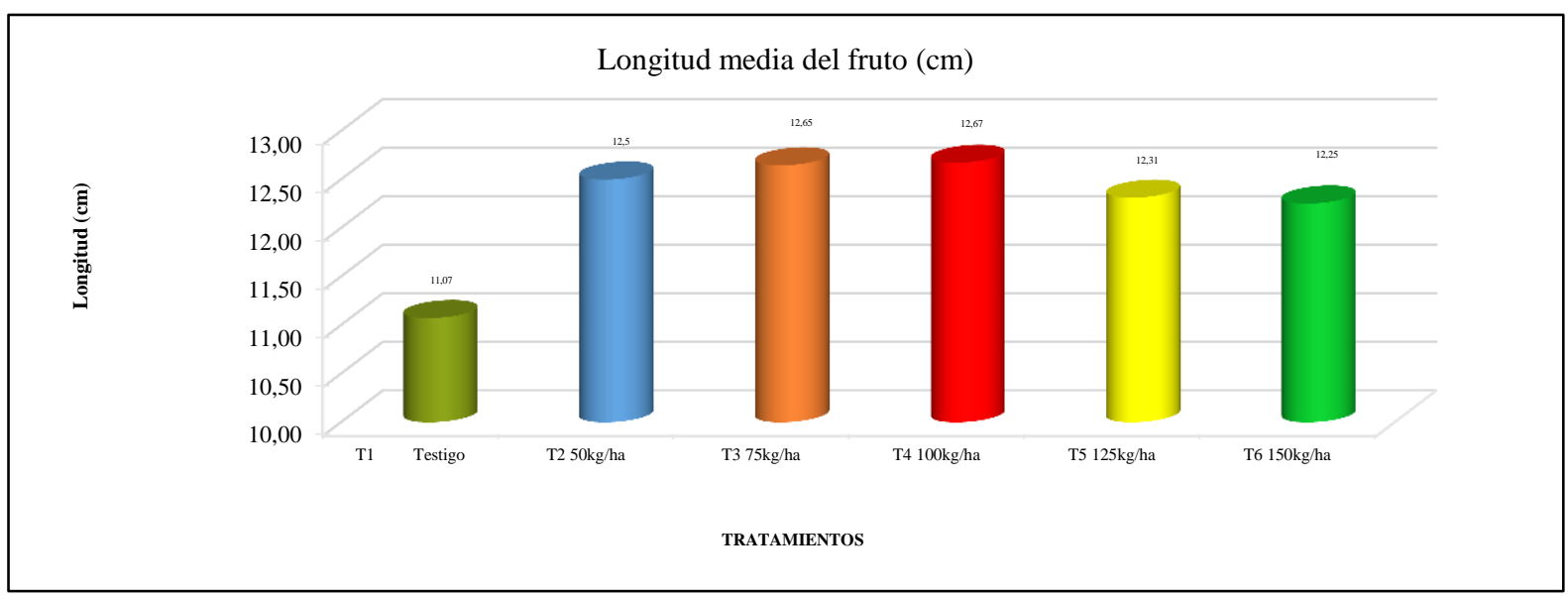

Figura 1. Valores promedios de longitud de frutos en la evaluación de diferentes niveles de fertilización potásica en el rendimiento y la calidad de frutos en el cultivo de pimiento. Pilar-Ñeembucú. 
En la Figura 1 se observa que el T1 (testigo) sin aplicación de fertilización potásica registró una media menor que los demás tratamientos con $11,07 \mathrm{~cm}$ y en cuanto a los tratamientos restantes con diferentes niveles de fertilización potásica, muestran un aumento en la longitud del fruto, proporcionando la mayor media el T4 (100 $\mathrm{kg} / \mathrm{ha}$ ), con $12,67 \mathrm{~cm}$, con una diferencia de 1,6 centímetros con respecto al tratamiento testigo y a partir de ahí un descenso con el T5 y T6.

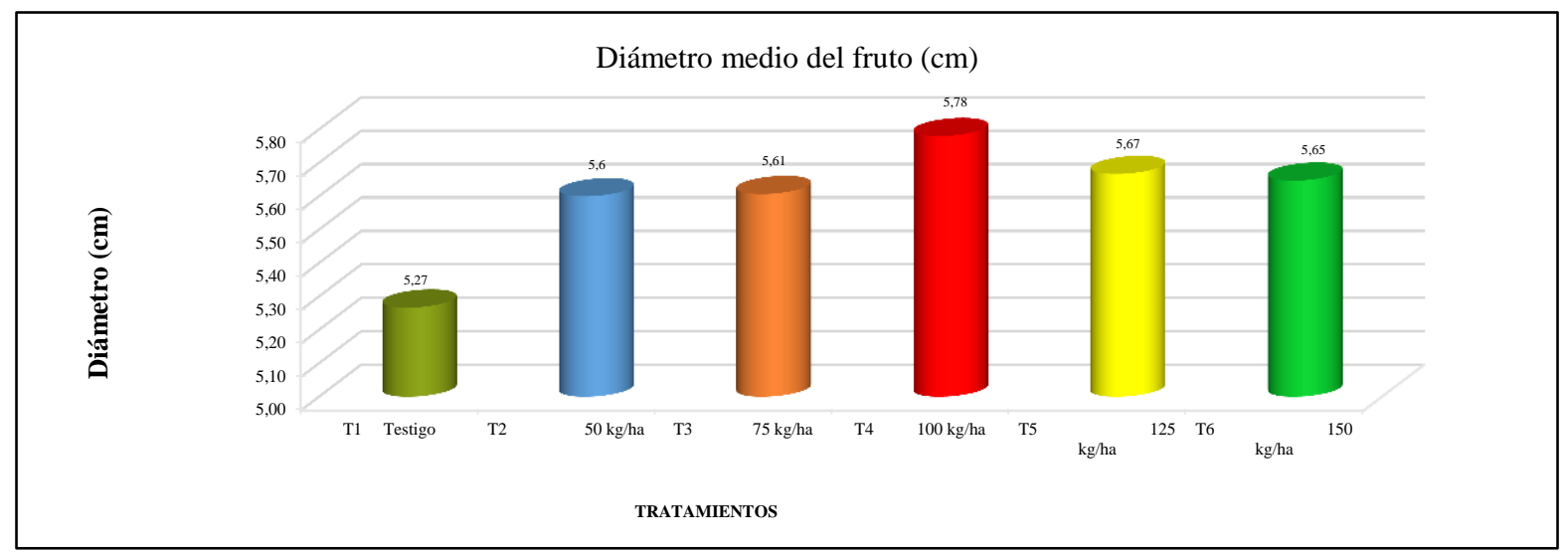

Figura 2. Valores promedios de diámetro de frutos en la evaluación de diferentes niveles de fertilización potásica en el rendimiento y la calidad de frutos en el cultivo de pimiento. Pilar-Ñeembucú.

En la Figura 2 se observar los valores promedios de diámetro de frutos por cada tratamiento efectuado, que fueron medidos de igual manera que la longitud con un calibre y con un total de 15 muestras de frutos. Se puede verificar que el T4 con la aplicación de $100 \mathrm{~kg} / \mathrm{ha}$ de cloruro de potasio se obtiene la mayor media con $5,78 \mathrm{~cm}$ con una diferencia alrededor de un milímetro sobre los demás tratamientos con fertilización potásica y una diferencia de 5 milímetros sobre el T1 (testigo) el cual presentó la menor media con $5,27 \mathrm{~cm}$.

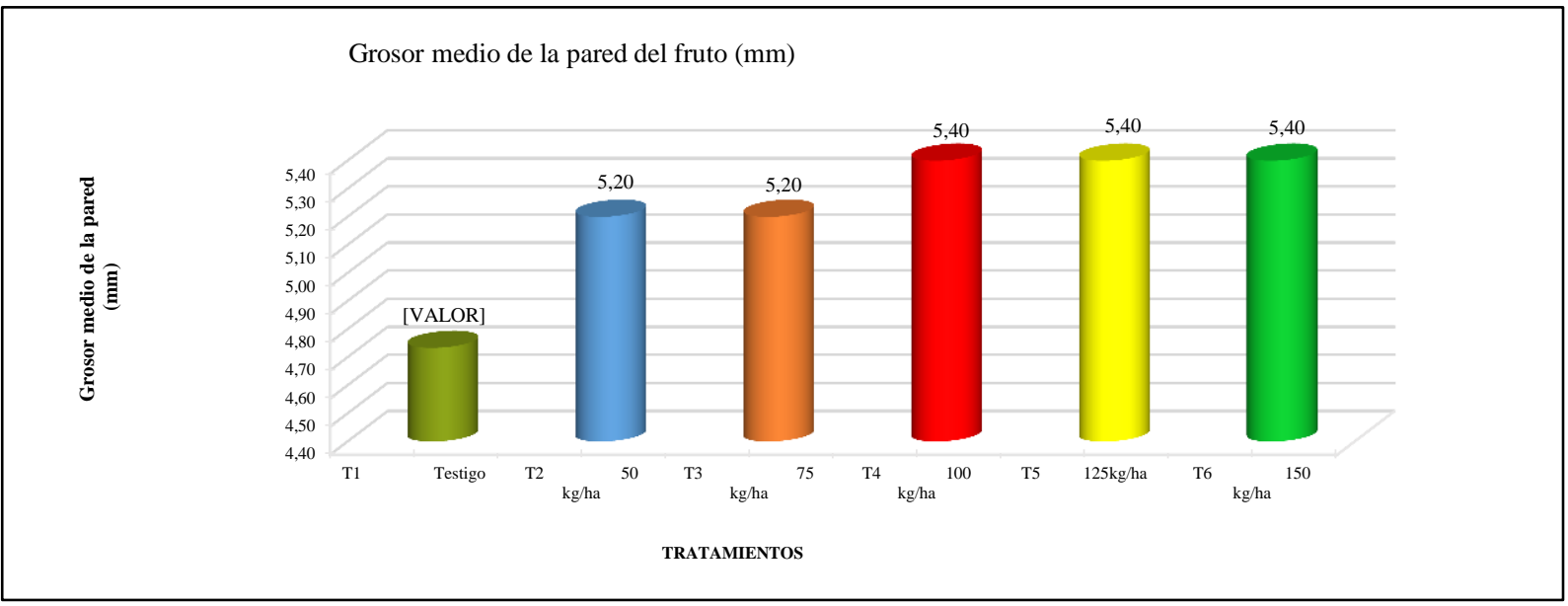

Figura 3. Valores promedios de grosor de la pared del fruto en la evaluación de diferentes niveles de fertilización potásica en el rendimiento y la calidad de frutos en el cultivo de pimiento. Pilar Ñeembucú. 
En en el apartado relacionado con la Figura 3 se muestran diferentes valores promedios en cuanto al grosor de la pared del fruto de los diferentes tratamientos aplicados la cual indica que el tratamiento sin aplicación de cloruro de potasio resultó con el menor valor con 4,73 mm, además se puede apreciar que el T4, T5 y T6 posee el mismo valor promedio de 5,40 $\mathrm{mm}$ siendo estas superiores al testigo. En esta figura se puede notar que la aplicación de fertilización potásica ayuda a aumentar el valor medio de grosor de la pared del fruto ya que el tratamiento testigo (sin aplicar) arrojo el menor promedio, comenzando a aumentar los valores con la aplicación del cloruro de potasio, pero los mismos no poseen diferencias mayores entre sí, y que a mayor concentración de cloruro de potasio no decrece su valor como ocurrió con los promedios de diámetro y longitud.

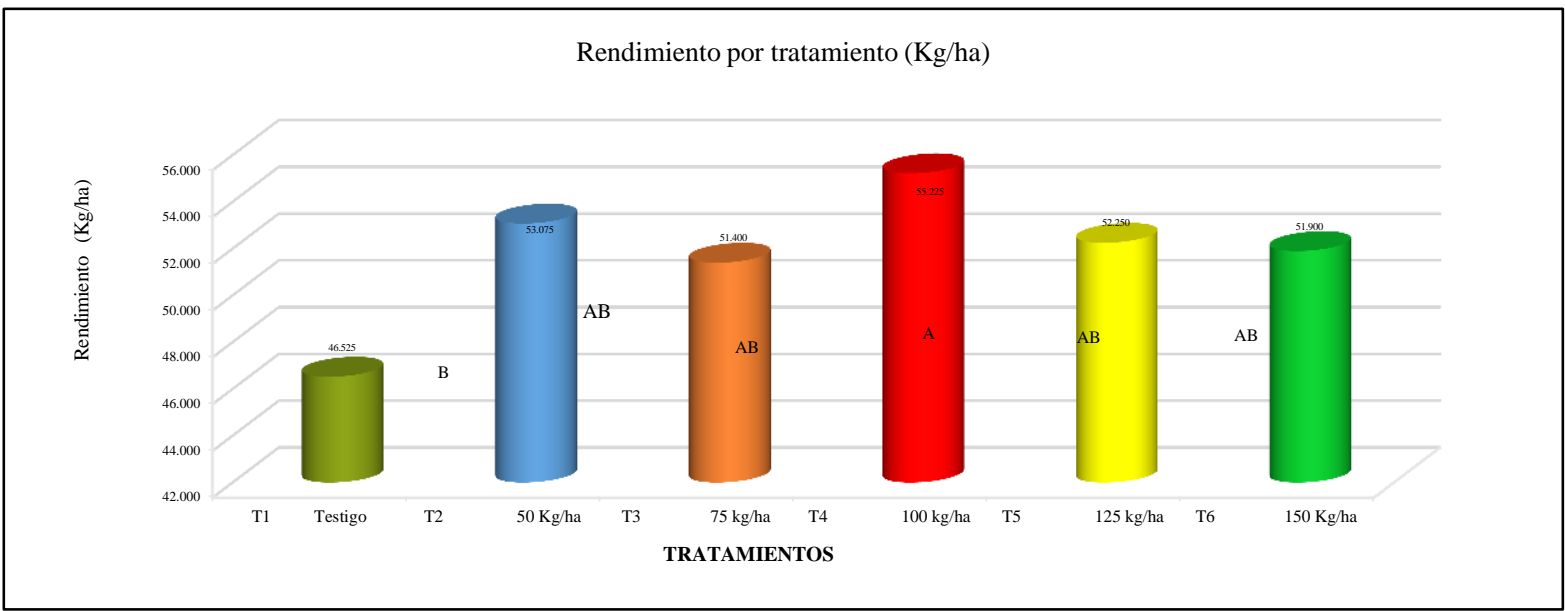

Figura 4. Valores promedio de rendimiento por tratamiento en la evaluación de diferentes niveles de fertilización potásica en el rendimiento y la calidad de frutos en el cultivo de pimiento. Pilar-Ñeembucú.

La Figura 4 muestra el rendimiento de pimiento en kilogramos por hectárea de los diferentes tratamientos, obteniéndose un mayor rendimiento con el T4 (100 kg/ha) con $55.225 \mathrm{~kg} / \mathrm{ha}$, siendo este el tratamiento con la fertilización recomendada por el análisis de suelo, y el menor rendimiento se obtuvo con el tratamiento testigo con $46.525 \mathrm{~kg} / \mathrm{ha}$. En cuanto al análisis de varianza con un nivel de significancia al 5\%, indica que no hay diferencia significativa entre los tratamientos, con un coeficiente de variación de 8,73\%. Una vez obtenido el análisis de varianza se procedió a someter a la prueba de comparación de Duncan en el cual se pudo verificar que el T4 presentó diferencia significativa sobre el T1 (Testigo) y que no existe diferencia significativa entre los tratamientos con fertilización potásica. 


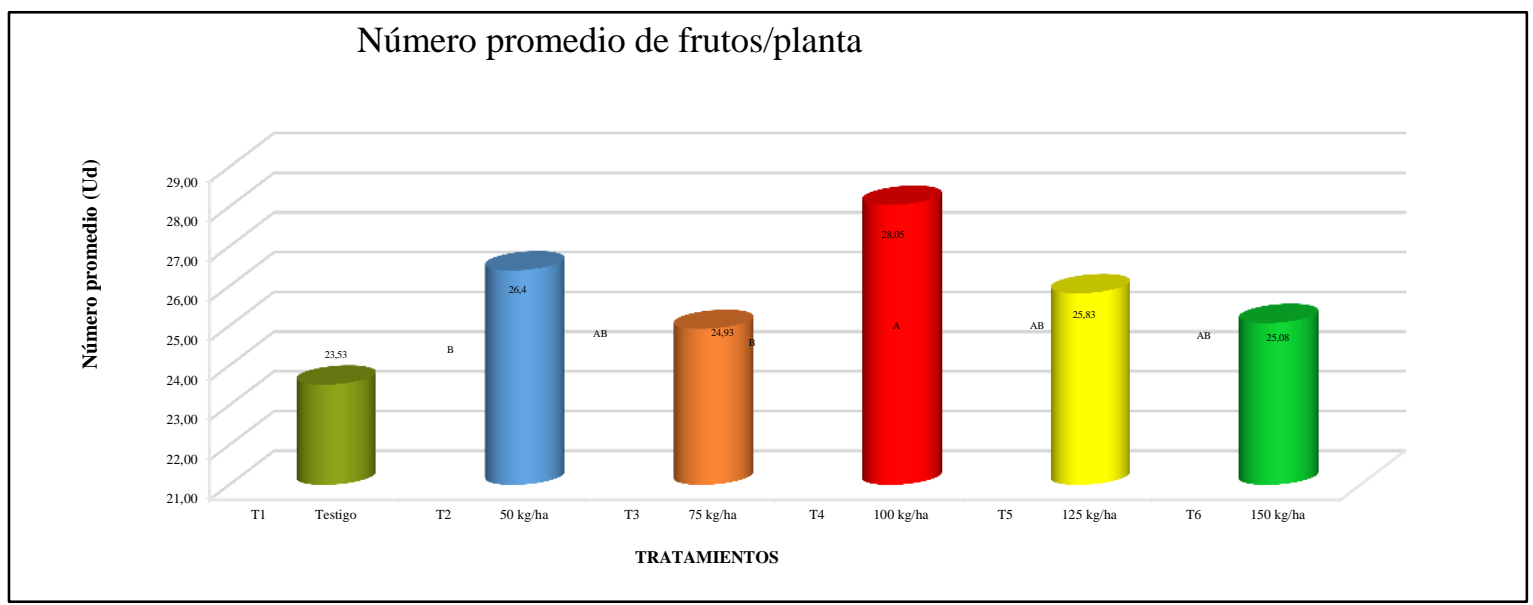

Figura 5. Valores promedios de números de frutos por planta en la evaluación de diferentes niveles de fertilización potásica en el rendimiento y la calidad de frutos en el cultivo de pimiento. Pilar- Ñeembucú.

Para obtener el número promedio de frutos por planta se contabilizo el total de frutos obtenidos en las 5 cosechas realizadas promediando con la cantidad de plantas que se tuvo en el área útil tal como se muestra en la Figura 5. El análisis de varianza con un nivel de significancia al 5\%, indica que no hay diferencia significativa entre los tratamientos, con un coeficiente de variación de 8,27 \%. Se observa los valores medios de número de frutos por planta en el cultivo de pimiento, donde se puede notar que el T4 con la aplicación de $100 \mathrm{~kg} / \mathrm{ha}$ de fertilización potásica resultó con mayor cantidad de frutos con un total de 28,05 frutos por planta, el cual, según la comparación de medias a través de la prueba de Duncan al 5\% presenta diferencia estadística significativa sobre el T3 (75 kg/ha), y sobre el T1 (testigo) sin fertilización potásica, que presentó la menor media con 23, 53 frutos por planta. Por lo tanto, el mayor número de frutos obtenido con el T4, influyó en la obtención de un mayor rendimiento en este tratamiento.

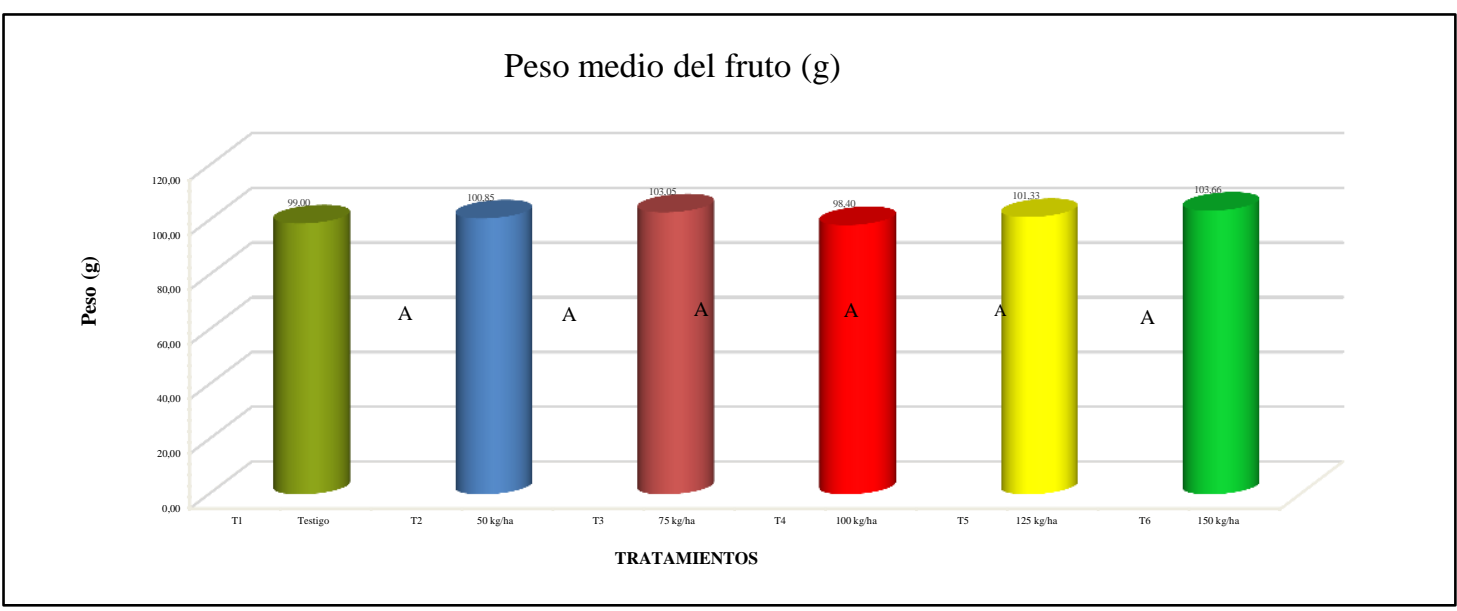

Figura 6. Valores promedios de peso de frutos, en la evaluación de diferentes niveles de fertilización potásica en el rendimiento y la calidad de frutos en el cultivo de pimiento. Pilar-Ñeembucú. 
De acuerdo a esta variable se puede observar que el T4 tuvo el menor peso promedio con 98,40 gramos $y$ valores superiores con los tratamientos y $\mathrm{T} 3$ con 103,66 y 103,05 gramos respectivamente. Aunque el T4 presentó un mejor rendimiento (kg/ha), no tuvo el mayor peso promedio de fruto; con lo que se puede verificar que el peso medio de frutos no influyó en la obtención de un mayor rendimiento, sino que esto se produjo por la mayor cantidad de frutos por planta. En el análisis de varianza con un coeficiente de variación de 8,73\% y el nivel de significancia al 5\%, al igual que la prueba de Duncan indican que no existe diferencia significativa entre los tratamientos.

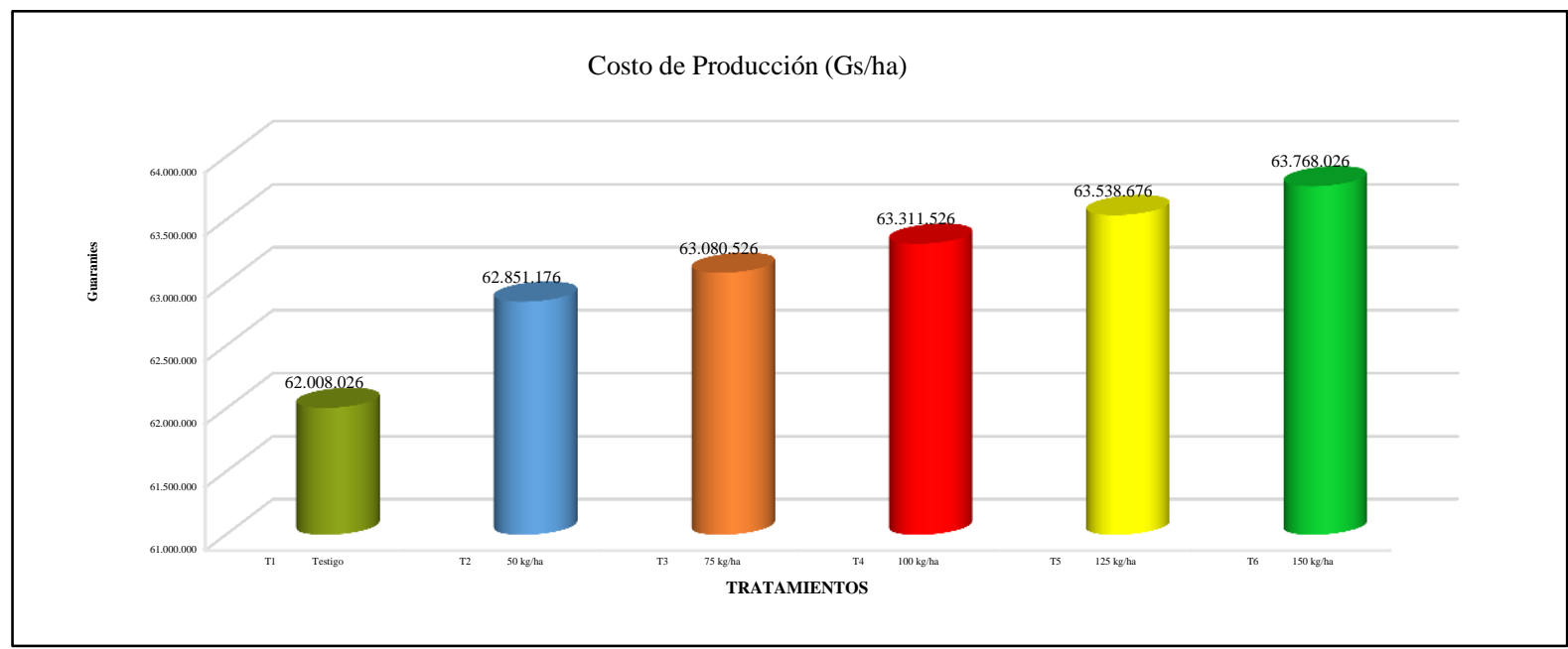

Figura 7. Costo de producción de los tratamientos en la evaluación de diferentes niveles de fertilización potásica en el rendimiento y la calidad de frutos en el cultivo de pimiento. Pilar-Ñeembucú.

En la Figura 7 muestra los costos de producción de los tratamientos, medidos en guaraníes por hectárea, donde se tuvo en cuenta los insumos, como la obtención de plantines, fertilizantes, plaguicidas entre otros, los mismos están en relación con la aplicación de los diferentes niveles de fertilización potásica, así también se valoró la mano de obra, teniendo en cuenta la preparación del terreno, el trasplante, los cuidados culturales y la cosecha. Se puede observar que el T1 tuvo el menor costo de producción con 62.008.026 Gs/ha, a partir del T2 existe un aumento en el costo de producción debido a la aplicación de fertilizante a base de potasio, donde el T5 y T6 tuvieron los costos de producción más elevados cuyos valores ascienden a 63.538.676 Gs/ha y 63.768.026 Gs/ha respectivamente, llegando al punto máximo con el T6 con la mayor dosis de aplicación de fertilizante potásica. 


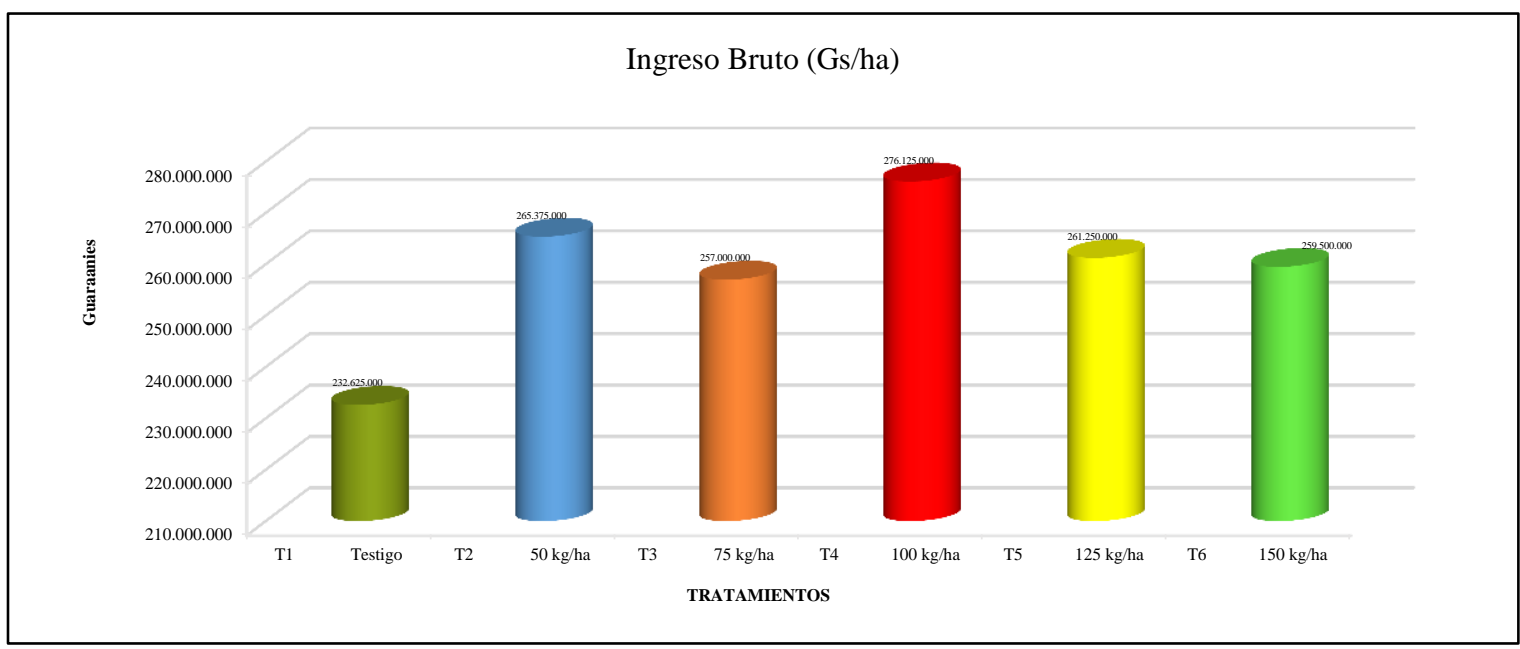

Figura 8. Ingreso Bruto por cada tratamiento en la evaluación de diferentes niveles de fertilización potásica en el rendimiento y la calidad de frutos en el cultivo de pimiento. Pilar-Ñeembucú.

En la Figura 8 se observa el ingreso bruto obtenidos del cálculo de rendimiento en kilogramos alcanzados en cada uno de los tratamientos, expresados en hectáreas, esto multiplicado con el precio del producto, evaluados a 5.000 Gs por kilogramo dichos resultados arrojaron que el mayor ingreso bruto en guaraníes se obtiene con el tratamiento con mayor rendimiento, el $\mathrm{T} 4$ con 276.125.000 Gs/ha y el menor ingreso bruto con el tratamiento testigo sin aplicación de fertilización potásica.

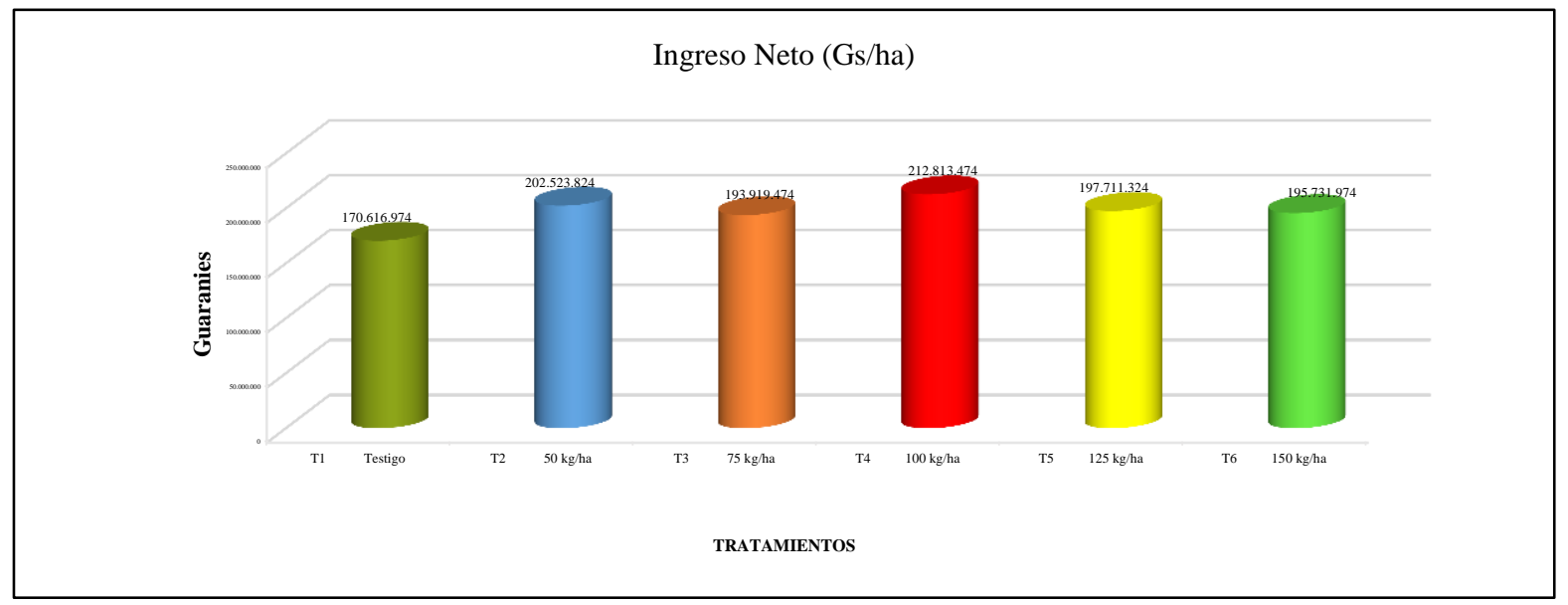

Figura 9. Ingreso Neto por cada tratamiento en la evaluación de diferentes niveles de fertilización potásica en el rendimiento y la calidad de frutos en el cultivo de pimiento. Pilar-Ñeembucú. 
En este apartado se puede observar el ingreso neto de los diferentes tratamientos expresados en guaraníes por hectárea, para lo cual se realiza el cálculo de diferencia entre el costo de producción y el ingreso bruto (Figura 9). Se puede verificar que con el tratamiento con la aplicación de fertilización potásica de acuerdo a la recomendación del análisis de suelo (T4) presenta un ingreso en guaraníes por hectárea mayor con respecto a los demás tratamientos con 212.813.474 Gs/ha. El tratamiento con el menor costo de producción el testigo sin la aplicación de fertilización potásica y de menor rendimiento fue quien arrojó con el menor beneficio en guaraníes por hectárea con 170.616.974 Gs.

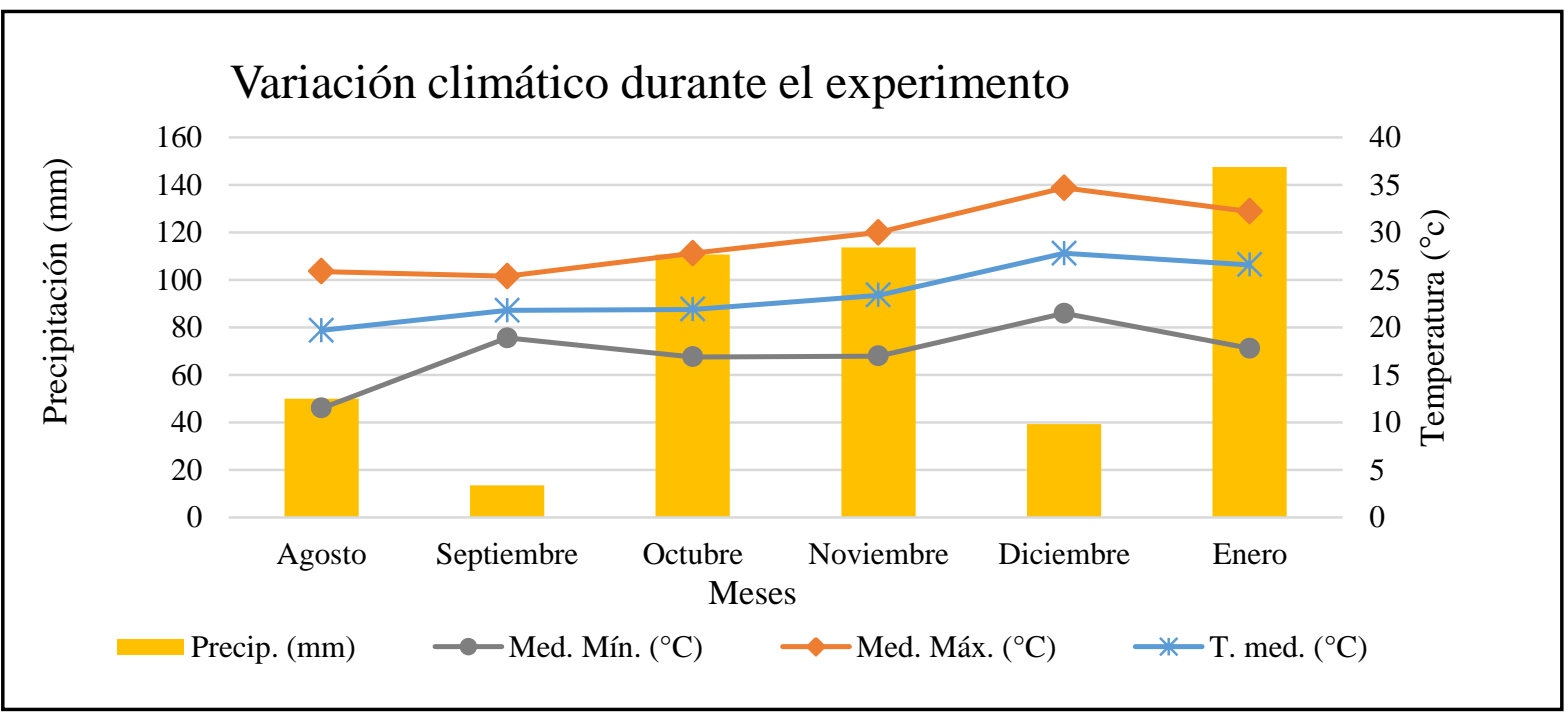

En la Figura 10 son registradas las variaciones de temperaturas medias, mínimas y máximas en grados centígrados suscitadas durante el periodo de duración del experimento. Las temperaturas reinantes durante la etapa inicial de los plantines no causaron mayores problemas debido que la germinación se llevó a cabo en Estufa de Germinación con temperaturas optimas y una vez germinadas las semillas se prosiguió al repique para luego ser acondicionados en un micro túnel donde mantenían temperaturas ideales para su crecimiento vegetativo hasta el momento del trasplante a la parcela experimental. Y para evitar quemaduras en los frutos de pimiento por las altas temperaturas de los meses de la cosecha noviembre, diciembre y enero se tomó la precaución de implementar mallas medias sombras de $35 \%$ para contrarrestar ese posible problema.

En cuanto a las precipitaciones, en los meses de septiembre y diciembre en donde se registraron la menor cantidad de lluvia caída, con $13,6 \mathrm{~mm}$ y $34,9 \mathrm{~mm}$, mientras que las mayores precipitaciones se constataron en los meses de octubre, con 110,8 mm, noviembre, con 113,8 $\mathrm{mm}$ y enero con $147,6 \mathrm{~mm}$ no ocasionando inconvenientes ya que se tomaron prevenciones ante una excesiva lluvia, realizando camellones altos para su trasplante evitando así el problema de encharcamiento, debido que los plantines no lo toleran, y en cuanto a la cantidad mínima 
de lluvia caída, se compensaron las necesidades de agua con abundante riego realizado con sistema de riego por goteo.

\section{Análisis de suelo}

El grado de acidez que presenta el suelo es muy ácido con 4,73 por lo cual se le aplicó cal agrícola para mejorar el $\mathrm{pH}$ del suelo y en cuanto al porcentaje de materia orgánica como bien se sabe el departamento de Ñeembucú se caracterizan por poseer suelos muy pobres que se puede evidenciar en este resultado con un porcentaje $0,17 \%$ por lo tanto, la aplicación de materia orgánica fue indispensable en la parcela experimental cuya fuente fue el estiércol vacuno a razón de 20 $\mathrm{Tn} / \mathrm{ha}$. Y con respecto a los macronutrientes presentes en el análisis también arrojaron índices muy bajos de manera que para obtener rendimientos satisfactorios fue fundamental la fertilización mineral con una recomendación acorde al resultado del análisis de suelo. El plan de fertilización de acuerdo a la recomendación del Ing Agr. Waldir Vera, docente de la Facultad de Ciencias Agrarias, Universidad Nacional de Asunción sede Santa Rosa-Misiones, fue de 120-120-100 kg/ha de $\mathrm{N}, \mathrm{P}_{2} \mathrm{O}_{5} \quad \mathrm{y} \quad \mathrm{K}_{2} \mathrm{O}$, respectivamente, esto para compensar la baja concentración de los macroelementos.

\section{CONCLUSIONES}

Bajo las condiciones en que se realizó este experimento y con base en los resultados obtenidos y los análisis realizadas, se concluye como sigue: El T4 con la aplicación de $100 \mathrm{~kg} /$ ha de fertilización potásica permite obtener mayor longitud y diámetro de fruto, aunque estas sean mínimas con los demás tratamientos, por su parte, en cuanto al grosor de pared de fruto a partir del T4 se obtuvo mayores promedios, el cual no tuvo diferencias con el T5 (125 kg/ha) y T6 (150 $\mathrm{kg} / \mathrm{ha}$ ).En lo referente a la coloración y forma comercial de los frutos se puede mencionar que no presentaron diferencias relevantes ya que los mismos presentaron similitudes en cuanto a su tamaño y forma como también en la coloración. En cuanto al peso promedio de frutos no hubo diferencias significativas entre los tratamientos, por lo tanto, esta característica no se vio afectada por los diferentes niveles de fertilización potásica. Con respecto al rendimiento, el T4 con 100 $\mathrm{kg} / \mathrm{ha}$ de fertilización potásica permitió obtener el mayor rendimiento, el cual presentó diferencia significativa sobre el testigo, según la prueba de Duncan, demostrando que la aplicación de fertilización potásica al cultivo de pimiento tiene un efecto positivo para mejorar la producción. Así también, el número de frutos por planta presentó diferencias significativas sobre el T1 y T3, con esta misma aplicación (100 kg/ha), la cual permite alcanzar un mayor beneficio neto.

\section{REFERENCIAS}

1. Enciclopedia virtual.EUMED.NET. Metodología Cuantitativa y Cualitativa. [En línea]. URL Disponible https://www.eumed.net/tesisdoctorales/2012/zll/metodologiainvestigacion.html\#: :text=Para\%20reforzar \%20este\%20criterio\%20M\%C3\%A9ndez

2. Reyes Castañeda. P. Diseño de Experimentos Aplicados. 2a ed. México. Trillas. 1980

3. Enciclopedia virtual.EUMED.NET. Metodología Cuantitativa y Cualitativa [en línea] URL disponible https://www.eumed.net/tesisdoctorales/2012/zll/metodologiainvestigacion.html\#: :text=Para\%20reforzar $\% 20$ este $\% 20$ criterio\%20M\%C3\%A9ndez 
4. Enciclopedia virtual.EUMED.NET. investigacion.html\#: :text=Para\%20reforzar Metodología Cuantitativa y Cualitativa [en \%20este\%20criterio\%20M\%C3\%A9ndez línea] URL disponible https://www.eumed.net/tesis5. Tamayo y Tamayo, M. El proceso de la doctorales/2012/zll/metodologiaInvestigación Científico. 3ra edición. México: LIMUSA. 1998. 